\title{
Avaliação de Desempenho de Tráfego IPTV sobre pDSL - Uma Abordagem baseada em Aferição.
}

\author{
Marcos César R. Seruffo ${ }^{1}$, Dário Russillo ${ }^{1}$, Diego L. Cardoso ${ }^{1}$, Marcelino S. Silva ${ }^{1}$, Lamartine V. de \\ Souza $^{2}$, Carlos R. Francês ${ }^{1}$, João C. W. A. Costa ${ }^{2}$, Jaume R. I Riu ${ }^{3}$
}

\begin{abstract}
Resumo - A tendência crescente de transmissão de tráfego triple play (voz, vídeo e dados) via internet é um fenômeno de amplitude mundial. Entretanto, para obter-se uma comunicação satisfatória, deve-se estudar o processo de maneira fim-a-fim. Em regiões com pouca infra-estrutura de telecomunicações, uma das preocupações recai sobre as tecnologias de acesso utilizadas. Este trabalho apresenta uma análise baseada em aferição de uma transmissão IPTV (Internet Protocol Television) gerada sobre duas tecnologias de acesso combinadas, PLC (PowerLine Communications) e ADSL2+ (Asymmetric Digital Subscriber Line) utilizando uma combinação de infra-estruturas de rede elétrica e de telefonia, denominada de pDSL, que se apresenta como uma combinação viável para regiões com pouca infraestrutura de comunicação disponível. Os resultados são avaliados com o intuito apresentar a viabilidade do ambiente de estudo proposto.
\end{abstract}

Palavras-chave -pDSL, IPTV, ADSL2+, PLC e análise experimental.

Abstract - The increasing trend of triple play traffic transmission (voice, video and data) over internet is a worldwide phenomenon. However, to get a satisfactory communication, we need study the process on end-to-end way. Into regions with poor infrastructure of telecommunications, one of the concerns is about used technologies of access. This work presents an analysis based on an IPTV (Television Internet Protocol) transmission generated on two combined technologies of access, PLC (PowerLine Communications) and ADSL2+ (Asymmetric Digital Subscriber Line), using a combination of electric network and telephony infrastructure, called of pDSL. This technology is a viable combination for regions with poor infrastructure of telecommunications. The results are evaluated with intention to present the viability of this kind of the telecommunication environment.

Keywords - pDSL, IPTV, ADSL2+, PLC and experimental analysis.

\section{INTRODUÇÃOO}

Com a evolução das redes de computadores, cujas capacidades de comunicação e garantias de transmissão vêm aumentando substancialmente, novas vertentes tecnológicas começam a ser estabelecidas no cenário mundial. A evolução da tecnologia da informação traz uma tendência de transposição de barreiras infra-estrutrais

\footnotetext{
${ }^{1}$ Laboratório de Planejamento de Redes de Alto Desempenho (LPRAD) Universidade Federal do Pará (UFPA) - Belém/PA - Brasil.

${ }^{2}$ Laboratório de Eletromagnetismo Aplicado (LEA) - UFPA - Brasil.

${ }^{3}$ Ericsson, Armborstvägen 14, P.O. Box 1505, Älvsjö, Sweden 12525.

\{seruffo, dario, diego, marcelino, lvsouza, rfrances, jweyl\}@ufpa.br, jaume.rius.i.riu@ericsson.com.
}

para que se possa ter resultados positivos. Com isso, algumas tecnologias vêm sendo desenvolvidas no sentido de acompanhar essa evolução latente, aumentando a capacidade de disseminação da informação.

Nesse sentido é de fundamental importância que as tecnologias utilizadas tenham certas características para prover uma real ampliação de serviços de cidadania e/ou inclusão digital/social. Essas características, como capilaridade abrangente, baixos custos e facilidade de implantação e uso, são importantes uma vez que alcançam regiões com pouca infra-estrutura disponível ao usuário final típico, as quais comumente possuem especificidades geográficas que dificultam a consolidação dessas soluções.

Assim, deve-se perseguir sempre as possíveis combinações de tecnologias que possam alargar as chances do sucesso daquelas de cunho social. Para tanto, é imprescindível valer-se de estudos a priori de tecnologias que apresentem relações custo/benefício favoráveis a determinadas soluções de telecomunicações. Neste sentido, tecnologias que utilizam infra-estrutura já existente para outros sistemas podem amenizar o impacto de implantação. As tecnologias de acesso ADSL2+ e PLC, por exemplo, tem a seu favor o fato de o ADSL2+ ser uma tecnologia já bastante consolidada no mercado, uma vez que utiliza a linha telefônica para transmissão de dados; e que o PLC possui uma grande capilaridade, sendo considerado como uma tecnologia ubíqua, já que faz usufruto da rede de energia elétrica para transmissão de dados. Assim, entende-se que a solução combinando PLC indoor com ADSL2+ como rede de acesso é uma das mais viáveis para promover ações de inclusão digital em regiões como a Amazônia.

Este artigo apresenta um estudo de caso de tráfego IPTV utilizando a combinação pDSL, com o intuito de apresentar medidas que comprovem a viabilidade da solução proposta. A escolha por IPTV se deve ao fato do forte apelo social e abrangência que a mídia TV possui no Brasil, chegando a atingir mais $90 \%$ dos domicílios brasileiros, segundo o IBGE [1].

$\mathrm{O}$ restante do artigo está dividido da seguinte forma. $\mathrm{Na}$ seção II são abordados os conceitos básicos de IPTV. Na seção III são apresentados as características básicas das tecnológicas de acesso ADLS2+, PLC e pDSL. Na seção IV são discutidos o cenário e a abordagem utilizada neste artigo. A seção V apresenta os resultados obtidos e a seção final mostra as conclusões obtidas. 


\section{IPTV}

IPTV é um serviço de internet bilateral de alta velocidade, que possui uma comunicação através de pacotes IP (Internet Protocol), enviando imagens com padrões de nível definidos e provendo o serviço de triple play, ou seja, é um serviço que combina voz, dados e vídeo sob um único canal de comunicação de banda larga, inclusive o envio de informação multimídia (streaming) [2], [3] e [4].

O serviço de IPTV faz uso de uma rede banda larga para entregar conteúdo de TV potencialmente acrescido de serviços interativos. As redes IP atuais oferecem um serviço de entrega de pacotes chamado de "melhor esforço", que não oferece garantias de desempenho para seus usuários, com isso pacotes podem ser perdidos no seu trajeto.

O retardo, a perda de pacotes e a capacidade de transmissão da rede são muito importantes para as aplicações de IPTV, uma vez que estas redes podem tolerar pequenas perdas de pacotes, em contrapartida impõem restrições severas de temporização ou de capacidade mínima de transmissão para garantir sua própria viabilidade.

\section{TECNOLOGIAS DE ACESSO}

Para o fornecimento do serviço de IPTV, faz-se necessária uma tecnologia que ligue o usuário final à rede de transmissão de dados (neste caso, chamada de tecnologia de última milha).

O acesso a serviços de comunicação normalmente é provido não por uma única tecnologia, mas sim por uma combinação delas, o que gera uma infinidade de problemas, tais como: diferenças entre larguras de bandas, dificuldades para garantir qualidade de serviço (QoS), confiabilidade e escalabilidade. Por conta disso, é fundamental que se tenha um conjunto de ferramentas de suporte à decisão para possibilitar planejamento de implantação das tecnologias existentes.

Atualmente existem várias tecnologias de última milha, sendo que estas podem ser divididas em duas classes: (a) tecnologias cabeadas: fibra ótica (para acesso e não para backbone); PLC; CATV (TV a cabo); xDSL e linha discada; (b) tecnologias de acesso sem fio (Wireless): satélites, sistemas celulares e redes locais sem fio (WLANs).

\section{A. ADSL2+}

Digital Subscriber Line é uma família de tecnologias que fornecem um meio de transmissão digital de dados, aproveitando a própria rede de telefonia que chega na maioria das residências. ADSL2+ advém de DSL, sendo que permite a transmissão de dados mais rápida que a convencional ADSL.
As tecnologias DSL possuem uma grande abrangência no cenário mundial, sendo consideradas como a tecnologia de acesso banda larga dominante não só na Europa, como também na América Latina e em países em desenvolvimento como a Índia [5], [6] e [7].

Na América Latina a tecnologia DSL é responsável por cerca de $77 \%$ de todo o acesso banda larga realizado, sendo que no Brasil este valor chega a $85 \%$. Em termos quantitativos, no final de 2005 havia quase 5.300.000 assinantes na América Latina de tecnologia ADSL [6].

Apesar de tais números, a penetração dos serviços banda larga nas residências da América Latina foi estimada em apenas $9 \%$ para o final de 2006, sendo projetada em $17 \%$ para o final de 2015 [6]. Desta forma, pode-se compreender que há um longo caminho a percorrer para a massificação dos serviços banda larga na América Latina.

\section{B. PLC}

As redes PLC utilizam a rede de distribuição de energia elétrica como meio de transmissão de sinais de comunicação. A tecnologia PLC possui a vantagem de ter uma infra-estrutura de alta capilaridade e custo associado relativamente baixo, mas possui algumas dificuldades [8]. O meio físico utilizado é bastante hostil para a transmissão de dados, visto que não foi projetado para este fim, pois há uma série de propriedades das redes de energia que influenciam negativamente na comunicação em alta velocidade, tais como: perdas no cabo, propagação em múltiplos caminhos e ruídos [8] [9].

Uma forma de reduzir o impacto do meio de transmissão na comunicação é a aplicação de métodos eficientes de modulação, como o OFDM (Ortogonal Frequency Division Multiplexing), além de mecanismos de correção de erro como o FEC (Forward Error Correction) e o ARQ (Automatic Repeat Request) [10].

A propagação do sinal através da linha de transmissão de energia elétrica provoca uma atenuação e um atraso no sinal, os quais aumentam com a distância e a freqüência. Esta atenuação depende da impedância característica e da constante de propagação das linhas de transmissão. De acordo com [11] [13], estes dois parâmetros são funções da resistência, condutância, indutância e capacitância, por unidade de comprimento, os quais dependem da frequiência.

A tecnologia PLC pode ser utilizada tanto para rede local como para tecnologia de acesso, sendo que a aplicação desta tecnologia como rede de última milha (outdoor) possui ainda outras barreiras a serem transpostas, como por exemplo, o assinante deste serviço tem uma interface com a rede de baixa tensão, que possui inúmeros transformadores ao longo de sua extensão. O sinal PLC deve fazer um bypass nestes equipamentos, visando manter a integridade das informações transportadas. Podem ser utilizados, por exemplo, repetidores PLC para evitar os transformadores ao longo do caminho, tipicamente em redes de baixa tensão. Mas dependendo da extensão da rede, o investimento na 
implantação do serviço pode aumentar substancialmente por conta desse inconveniente.

\section{C. $p D S L$}

Powerline Digital Subscriber Line é uma solução de comunicação de dados em alta velocidade que combina duas tecnologias de transmissão de dados: ADSL e PLC. O ADSL como tecnologia de acesso banda larga, por estar mais consolidada no mercado e a tecnologia PLC no ambiente indoor, para distribuição do sinal, devido sua facilidade de implantação, visto que todas as tomadas passam a ser pontos de acesso em potencial, bastando, para isso, conectar um modem PLC em cada uma delas, montando assim uma rede local.

$\mathrm{O}$ seu funcionamento norteia-se basicamente em fornecer múltiplos links banda larga de alta velocidade sobre linhas de força (power lines) em um ambiente indoor [14].

Uma rede pDSL consiste de uma configuração de links de alta velocidade entre um Gateway PLC e múltiplos dispositivos pDSL. Tais dispositivos utilizam procedimentos de treinamento de canal para alocar largura de banda para cada link virtual. Em [15] foi realizado um estudo que descreve os critérios para desempenho ótimo de alocação de largura de banda para links em redes pDSL.

Para interligação entre redes utilizam-se gateways pDSL, que possuem função de central de comunicação e unidade de coordenação de uma rede pDSL, além de interface para infra-estrutura de comunicações externas (Figura 1).

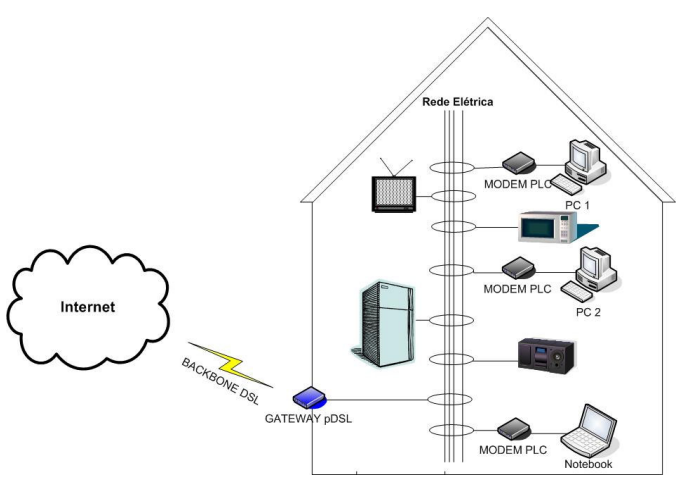

Figura 1: Estrutura de Serviços pDSL [10].

Em uma rede pDSL indoor existem duas categorias de dispositivos que são conectados ao seu ponto terminal (tomada AC), que são: o equipamento digital que requer alta velocidade na transmissão (conhecidos como dispositivos pDSL), tais como computadores, impressoras e TVs; e dispositivos que não fazem uso de tais serviços, embora utilizem a mesma linha de força.

A Figura 1 ilustra o exemplo de uma rede utilizando as tecnologias pDSL para ambiente indoor, contendo dispositivos pDSL e não pDSL conectados à rede elétrica. $\mathrm{O}$ gateway pDSL interliga a rede indoor com uma tecnologia de última milha existente (xDSL ou wireless, por exemplo).

\section{ESTUDO DE VIABILIDADE BASEADO EM AFERIÇÃO DO SISTEMA}

\section{A. Cenários}

Para análise deste experimento, foram utilizados dois cenários montados no LabIT (Laboratório de Inovação Tecnológica em Telecomunicações) da UFPA. O primeiro cenário, mostrado na Figura 2, é composto de DSLAM / EDA (rede xDSL), estação base PLC, modems PLC e um gerador de tráfego (AX-4000), capaz de gerar diversos tipos de tráfego (UDP e TCP), possibilitando assim um estudo mais aprofundado do comportamento da tecnologia pDSL em situações de alta carga.

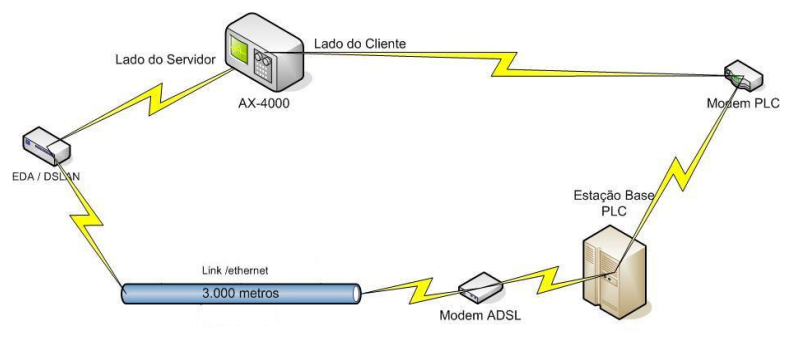

Figura 2: Cenário do experimento I.

O segundo cenário, mostrado na Figura 3, é típico de transmissão IPTV. Neste sistema, há máquinas comunicantes trafegando o triple play. O cenário de testes utilizado é composto de modems ADSL, modems PLC, DSLAM, cabos de telecomunicações, estação PLC, analisador de protocolo, gerador de ruído e computadores cliente e servidor.

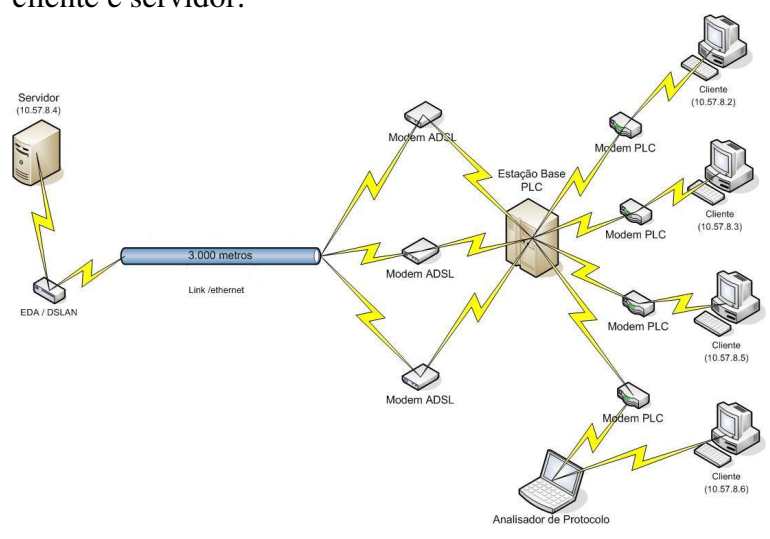

Figura 3: Cenário do experimento II.

\section{B. Descrição do experimento}

Nos dois cenários foi utilizado o conjunto DSLAM / EDA (Ethernet DSL Access) que compõe o equipamento existente no lado da central telefônica, permitindo assim a comunicação de dados via um enlace ADSL2+. 
Como tecnologia de última milha, escolheu-se utilizar cabos metálicos reais, os quais estão dispostos ao redor do prédio do LEA/UFPA (Laboratório de Engenharia Elétrica e Computação).

Para determinação do comprimento dos cabos, utilizouse a norma brasileira padrão Telebrás 225-540-788 de abril de 1997, a qual propõe a distância de 3.000 metros para um enlace de testes.

Para realizar a comunicação PLC, foi utilizada a própria rede elétrica do prédio do LEA/UFPA, nos quais os modens foram distribuídos entre diferentes pontos do laboratório.

Para geração do fluxo, no primeiro cenário, foi utilizado o AX/4000®, um equipamento desenvolvido pela Spirent Communications que possui a capacidade de gerar e analisar tráfego TCP/IP, no qual foi gerado um fluxo contínuo de pacotes TCP com tamanho fixo de 1000 bytes. Além da geração de tráfego, o AX/4000 pode realizar a emulação de modems ADSL2+, permitindo assim uma análise mais detalhada do desempenho de um sistema DSL.

No canário dois, um computador conectado ao DSLAM foi responsável por gerar fluxos de vídeo que serão distribuídos através de multicast entre os clientes. Para geração de tráfego de dados, utilizou-se um benchmark chamado IPERF. Trata-se de um software livre (versões para linux, Unix e Windows), utilizado para realizar acessos continuamente tanto TCP (simulando um HTTP), como UDP - disponível em [16].

$\mathrm{Na}$ geração de tráfego de vídeo, utilizou-se o VLC (VideoLAN Client), o qual é um reprodutor multimídia que suporta vários formatos de vídeo, além de vários protocolos de streaming [17]. Para o teste com vídeo, foi configurado o VLMS (VideoLAN MiniServer), servidor de streaming do VLC, que faz multicast, enviando a uma taxa de 1 Mbps para as três máquinas (vídeo com codec MPEG2) [17].

Para o transporte de voz, foi utilizado o Callgen, que é uma ferramenta VoIP desenvolvida pelo projeto OpenH232 e está disponível em [18], largamente utilizada para testes [19].

Um analisador de protocolo RADCOM® [20] também foi utilizado com a função de filtrar os pacotes que irão trafegar na rede, isolando fluxos específicos para geração de medidas de desempenho.

\section{Medidas para análise de desempenho}

Para um estudo apropriado de comparação entre as tecnologias utilizadas, foram feitas análises sobre as medidas de: atraso, jitter, MOS e perda de pacotes.

Todas as métricas relativas à transmissão de voz foram capturadas pelo MediaPro, um módulo pertencente ao analisador de protocolos RADCOM, responsável por capturar e avaliar todas as chamadas VoIP realizadas, informando MOS (Mean Opinion Score), jitter, perda de pacotes, dentre outras.

\section{RESULTADOS}

Os resultados foram obtidos e analisados individualmente para cada experimento realizado, conforme descrito anteriormente. Ambos cenários foram realizados estudos no sentido de downstream e upstream.

\section{A. Cenário I}

Neste cenário utilizou-se o equipamento AX-4000 para gerar um tráfego de dados TCP, com pacotes de tamanho fixo aproximadamente 1000 bytes. O fluxo gerado foi inserido continuamente no cenário, ocupando o máximo possível de banda (8Mbps de downstream e $800 \mathrm{Kbps}$ de upstream), simulando assim uma situação de alta carga, estressando ao máximo as tecnologias utilizadas.

Utilizou-se um filtro de linha e um carregador de pilhas como meios geradores de ruídos na rede PLC. Estes dispositivos foram utilizados em outros testes PLC [21] e [22]. As tabelas I e II apresentam os resultados obtidos para downstream e upstream respectivamente.

TABELA I

Avaliação de desempenho para o downstream (cenário I).

\begin{tabular}{|c|c|c|c|}
\hline & Sem ruído & Filtro de linha & Carregador \\
\hline Pacotes / s & 988 & 978 & 188 \\
\hline $\begin{array}{c}\text { Atraso } \\
\text { Médio }(m s)\end{array}$ & 16,055 & 16,754 & 16,058 \\
\hline Banda $(M b p s)$ & 7,907 & 7,823 & 1,507 \\
\hline $\begin{array}{c}\text { Taxa de pacotes } \\
\text { Perdidos }\end{array}$ & $8,18 \times 10^{-6}$ & $1,18 \times 10^{-4}$ & 0,355 \\
\hline
\end{tabular}

Percebe-se que com o filtro de linha inserido, quando o carregador de pilhas é colocado, ocorre uma grande perda na capacidade de transmissão, sendo que isso se deve ao fato do carregador possuir características de um transformador, conforme descrito em [21].

TABELA II

Avaliação de desempenho para o upstream (cenário I).

\begin{tabular}{|c|c|c|c|}
\hline & Sem ruído & Filtro de linha & Carregador \\
\hline Pacotes / s & 99 & 99 & 20 \\
\hline $\begin{array}{c}\text { Atraso } \\
\text { Médio (ms) }\end{array}$ & 43,083 & 43,344 & 53,543 \\
\hline Banda (Mbps) & 0,7939 & 0,7937 & 0,16 \\
\hline $\begin{array}{c}\text { Taxa de pacotes } \\
\text { perdidos }\end{array}$ & $7,17 \times 10^{-4}$ & $9,95 \times 10^{-4}$ & $4,33 \times 10^{-2}$ \\
\hline
\end{tabular}

Para o caso do tráfego upstream observa-se em linhas gerais um comportamento similar ao descrito anteriormente. 
Pode-se entender que o meio utilizado é bastante suscetível a ruídos, e que a introdução do carregador de pilhas no sistema resulta numa forte influência na comunicação, diminuindo a vazão em $20 \%$ e a taxa da perda de pacotes aumenta de um em um milhão para quatro em cem aproximadamente.

Em ambos os casos, o atraso médio não é tão influenciado pelas interferências.

Apesar disto e da grande distância fim a fim $(3000 \mathrm{~m})$ utilizada neste sistema a comunicação ainda é viável. Deve-se levar em conta que o sistema está em condições extremas de estresse, já que se está transmitindo um fluxo contínuo ocupando toda a banda disponível para tráfego.

\section{B. Cenário II}

A Tabela III apresenta um comparativo dos resultados obtidos durante cada uma das cinqüenta chamadas VoIP realizadas, estas chamadas foram feitas com e sem tráfego de background. Introduziu-se um filtro de linha na rede, com o intuito de se analisar o impacto de outros elementos pDSL na rede PLC.

TABELA III

Avaliação de desempenho para o upstream (cenário II).

\begin{tabular}{|c|c|c|c|c|}
\hline & \multicolumn{2}{|c|}{ VoIP } & \multicolumn{2}{c|}{ IPTV } \\
\hline Ruído & Sem & Aplicado & Sem & Aplicado \\
\hline MOS & 1,194 & 1,145 & 1,196 & 1,165 \\
\hline Banda (kbps) & 128,24 & 121,43 & 128,79 & 125,36 \\
\hline $\begin{array}{c}\text { Atraso de } \\
\text { resposta (s) }\end{array}$ & 3,98 & 4,45 & 6,57 & 4,97 \\
\hline Jitter (ms) & 11,8 & 12,1 & 11,6 & 11,9 \\
\hline
\end{tabular}

A Figura 4 mostra o comportamento da perda de pacotes, onde se nota que as transmissões na presença de ruído obtiveram um percentual médio maior de perda de pacotes, ficando em aproximadamente $35,83 \%$ de perda para transmissões somente de voz e $33,77 \%$ de perda para transmissões de voz com tráfego IPTV de fundo. A perda média de pacotes para transmissões sem ruído oscilou entre $31,67 \%$ e $31,59 \%$, sem tráfego e com tráfego IPTV de fundo, respectivamente.

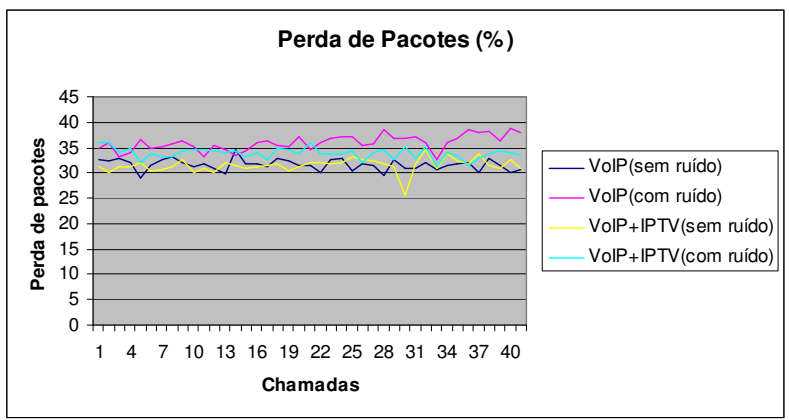

Figura 4: Perda de pacotes.
Através da Figura 4 fica claro que quando o ruído é inserido no sistema há uma queda no MOS, na largura de banda, um aumento no atraso do VOIP e um aumento no Jitter. Levando-se em conta que a análise é feita de uma aplicação em tempo real, qualquer diferencial obtido terá aplicação direta na transmissão das informações. A Tabela IV apresenta as medidas de downstream para o cenário II.

TABELA IV.

Avaliação de desempenho para o downstream (cenário II).

\begin{tabular}{|c|c|c|c|c|}
\hline & \multicolumn{2}{|c|}{ VoIP } & \multicolumn{2}{c|}{ IPTV } \\
\hline Ruído & Sem & Aplicado & Sem & Aplicado \\
\hline MOS & 4,2 & 4,2 & 4,2 & 4,2 \\
\hline Banda (bps) & 81678,71 & 81662,27 & 81652,08 & 81684 \\
\hline $\begin{array}{c}\text { Atraso de } \\
\text { resposta (s) }\end{array}$ & 130,7 & 138,8 & 1607,833 & 4094,182 \\
\hline Jitter (ms) & 0 & 0,6 & 4 & 1,4 \\
\hline Chamadas(60) & 60 & 58 & 54 & 45 \\
\hline
\end{tabular}

Apesar da grande distância utilizada neste sistema, a comunicação ainda é viável, apesar de apresentar níveis inferiores a sistemas correlatos em condições melhores. Entretanto, é imperativo levar-se em conta que o sistema está em condições extremas de estresse, já que está sendo transmitido tráfego IPTV, que se trata de uma aplicação que sistematicamente costuma valer-se de grande parte da largura de banda disponível.

Ressalta-se, portanto, que a estratégia de utilizar o "pior caso" valida a possbilidade dos demais tráfegos "best effort" (por exemplo, email).

\section{CONCLUSÃO}

A utilização da infra-estrutura existente na residência do usuário, além de agregar baixos valores na aquisição do serviço, atinge uma gama significativa de usuários finais potencialmente disponíveis para receber o serviço. Esse fator pode ser o diferencial entre prover-se ou não um determinado serviço em regiões com pouca infra-estrutura de comunicações (como é o caso da Amazônia). Este trabalho apresentou os resultados de uma análise experimental para transmissão banda larga de tráfego IPTV na presença ou na ausência de ruído, utilizando para transmissão uma rede indoor PLC e como solução de última milha a tecnologia ADSL2+ (da família xDSL).

Os resultados apresentados para os experimentos IPTV levam a concluir que um link de $800 \mathrm{kbps}$ como solução upstream de última milha (utilizando ADSL2+) em conjunto com uma rede pDSL indoor apresenta resultados satisfatórios para transmissão banda larga.

Os resultados desfavoráveis observados para o upstream no cenário II podem ser explicados pelo comprimento utilizado no enlace ADSL2+ de teste. A distância utilizada 
foi baseada na norma Telebrás 225-540-788 de abril de 1997, a qual se mostra inadequada como referência para tais testes, já que normas mais atuais [23] indicam uma distância máxima para enlaces de teste na ordem de 2500 $\mathrm{m}$. Observa-se assim que os valores de referência das normas tomadas como base deste experimento, quando submetidos a situações externas como as da Amazônia apresentam algumas inconformidades e que obrigatoriamente devem ser consideradas em projetos de redes de acesso, sob pena de inviabilizar o tráfego de determinadas aplicações com pesados requisitos de QoS.

Testes estão em andamento com o objetivo de qualificar um enlace pDSL em função do comprimento do backbone ADSL2+, de tal forma que seja possível estabelecer uma relação entre distância versus qualidade de serviço do sistema.

\section{AGRADECIMENTOS}

Os autores gostariam de agradecer ao Centro de Pesquisa e Desenvolvimento da Ericsson Telecomunicações Brasil, ao Departamento de Engenharia Elétrica e Computação da Universidade Federal do Pará, ao $\mathrm{CNPq}$ (Conselho Nacional de Desenvolvimento Científico e Tecnológico), a CAPES (Coordenação de Aperfeiçoamento de Pessoal de Nível Superior) e ao projeto PROCAD (Programa de Cooperação Acadêmica).

\section{REFERÊNCIAS BIBLIOGRÁFICAS}

[1] Extraído de http://www.ibege.gov.br acessado em 05/05/2007.

[2] Seong, H. K. and Gil, H. L. (2006). "The Study on SLA Metrics and System Architecture for IP TV”. In: The 8th International Conference on Advanced Communication Technology (ICACT2006), Gangwon-Do, Korea.

[3] Al-Hezmi, A.; Rebahi Y.; Magedanz T.; Arbanowski S. "Towards an Interactive IPTV for Mobile Subscribers". In: ICDT/IEEE International Conference on Digital Telecommunications.2006.

[4] Wu, J., Peng, K., Lu, M. Lin, C., Cheng, Y., Huang, P., Yao, J., Cheng, H. H. (2006) "HotStreaming: Enabling Scalable and Quality IPTV Services”, In: IPTV Workshop, International World Wide Web Conference. May 23, Edinburgh, Scotland, United Kingdom.

[5] Olsen, B.; Katsianis, D.; Varoutas, D.; Stordahl, K.; Harno, J.; Elnegaard, N.; Welling I.; Loizillon, F.; Monath, T.; Cadro, P. "Technoeconomic Evaluation of the Major Telecommunication Investment Options for European Players", IEEE Network, vol. 20, issue 4, pp.6-15, July/August 2006.

[6] Arenas, D.; Caldas, C.; Ramundo, C.; Vargas, S.; Hostos, L. "Challenges to expanding Fixed Broadband Services in Latin America”, White Paper, Alcatel Telecommunications. September 2006.

[7] Faudon, V.; Vleeschauwer, D.; Festraets, E.; Ross, P. . "End-User Services for Broadband uptake in High-Growth Economies”, White Paper, Alcatel Telecommunications. September 2006.

[8] Meng, H. e Guan, Y. L. "Modeling and Analysis of Noise Effects on Broadband Power-Line Communications", In: IEEE Transactions on Power Delivery, Vol. 20, No. 2, Abril 2005.

[9] Zimmermann, M., Dostert, K. "Analysis and Modeling of Impulsive Noise in Broad-Band Powerline Communications", In: IEEE Transactions on Electromagnetic Compatibility, Vol. 44, No. 1, Fev. 2002.
[10] Hrasnica, H., Haidine, A., Lehnert, R. "Broadband Powerline Communications Networks - Network Design”, John Wiley \& Sons, Inc, 2004.

[11]Zimmermann, M., Dostert, K. The low voltage power distribution network as last mile access network-signal propagation and noise scenario in the HF-range" In: AEÜ Int. J. Electron. Commun., Vol. 54 , No. $1,2000$.

[12]Andreou, G. T., Manitsas, E. K., Labridis D. P., et al “Finite element characterisation of $L V$ power distribution lines for high frequency communications signals", In: $7^{\circ}$ International Symposium on Power-Line Communication and its Applications (ISPLC), Kyoto, Japão, 2003.

[13]Hooijen, O. "A channel model for the residential power circuit used as a digital communications medium" In: IEEE Transactions on Electromagnetic Compatibility, Vol. 40, No. 4, November 1998.

[14] Antonakopoulos, T.; Papandreou, N. "Subchannels Allocation on Multiple pDSL Lines". Power Line Communications and Its Applications, International Symposium on.2005.

[15]Anastasiadou, D.; Antonakopoulos, T. "Broadband Communications in the Indoor Power Line Environment: The pDSL Concept". n Proc. ISPLC'04, Zaragoza, Spain, March 2004, pp. 334--339. (2004). Extraído de http://citeseer.ist.psu.edu/anastasiadou04broadband.html acessado em 28/04/2007.

[16]Iperf - The TCP/UDP Bandwidth Measurement Tool. Extraído de http://dast.nlanr.net/Projects/Iperf/, acessado em 02/05/2007.

[17]VLC (2007). "VLC Media PLayer". Disponível em www.videolan.org/vlc, acessado em 10/01/2007.

[18]OpenH323. (2007) Disponível em http://www.openh323.org/, acessado em 10/01/2007.

[19]Mann, I.; McLaughlin, S.; Henkel, W.; Kirkby, R.; Kessler, T. "Impulse Generation with Appropriate Amplitude, Length, InterArrival, and Spectral Characteristics", IEEE Journal on Selected Areas in Communications. Vol. 20, n. 5, pp. 901-912, June 2002.

[20]Radcom. (2007) "The State of Art". Disponível em http://www.radcom.com/, acessado em 01/02/2007.

[21]SOUZA, Jorge Antonio Moraes de; SILVA, Marcelino Silva da; FRANCES, Carlos Renato Lisboa; COSTA, João Crisóstomo Weyl Albuquerque da; SEGATTO, Marcelo E V; ANTÔNIO, Flávio R; RODRIGUES, Gabryella, "A feasibility study of powerline communication technology for digital inclusion in Brazilian Amazon". In: SPIE OPTICS EAST. 2006, Boston-USA.

[22]Souza, J. A. M., Silva, M. S., Francês, C. R. L., Costa, J. W., Segatto, M. E. V., Antônio, F. R., Vijaykumar, N. L. "A Feasibility Study of PLC Technology for Digital Inclusion”. VI International Telecommunications Symposium (ITS2006), Fortaleza, Brazil, 2006.

[23] TR-100, ADSL2/ADSL2plus Performance Test Plan, DSL Forum, March 2007 